\title{
Case Report -Peripheral Ossifying Fibroma with Congenital Heart Disease
}

\section{Amitha HA*, Panchakshari Prasanna B K, Akshatha B S, Kiran Y C and Sangeetha S Patel}

Paediatric and Preventive Dentistry Rajiv Gandhi University of Health Sciences RGUHS Bangalore, India

*Corresponding Author: Amitha HA, Paediatric and Preventive Dentistry Rajiv Gandhi University of Health Sciences RGUHS Bangalore, India.

Received: October 09, 2019; Published: October 28, 2019

DOI: $10.31080 /$ ASDS.2019.03.0678

\begin{abstract}
Peripheral ossifying fibroma (POF) is one of the inflammatory reactive hyperplasia of gingiva. The gingiva is often the site of localized growths that are considered to be reactive rather than neoplastic in nature. POF is one such lesion which constitutes about $9 \%$ of gingival growth. The size of the lesion is usually small located in the anterior maxillary region. In this case report we present a 15 year old medically compromised boy with POF on the maxillary anterior palatal region.

Keywords: Peripheral Ossifying Fibroma, Irritational Fibroma, Laser
\end{abstract}

\section{Introduction}

Peripheral ossifying fibroma (POF) is a soft tissue growth that is seen on the interdental papilla. It can be pedunculated or broad based, which could be smooth surfaced and varies from pale pink to cherry red in colour. It is comprises about $9 \%$ of all gingival growths and to arise from the gingival corium, periosteum, and the periodontal membrane. It has also been reported that it represents a maturation of a pre-existing pyogenic granuloma or a peripheral giant cell granuloma [1].

\section{Clinical report}

A 15 year old boy reported to V S Dental college and Hospital to Department of paediatric dentistry with a chief complaint of small soft tissue growth in the palate, behind the lateral incisors. Intra oral examination revealed a pedunculated, pale pink in colour, firm and resilient mass on the palatal aspect of the maxillary right anterior tooth region, extending from lateral incisors to the mesial aspect of premolars. It measured $1.5 \mathrm{~cm}$ mesiodistally and and $1 \mathrm{~cm}$ buccopalatally. The surface of the lesion was smooth and intact without any ulceration [Figure 1]. History revealed that the lesion started growing on its own 6 months back. The lesion becomes painful on chewing food and subsides on its own. Intraoral examination also shows lower anterior crowding and deep bite
[Figure 2]. Medical history revealed that patient has mitral valve prolapse and is on medication. Patients father also gives the same history of congenital heart disease. Patients previous medical report revealed that patient had palpitations, shortness of breadth, frequent cough, migraine headache and chest discomfort. The radiograph revealed only a soft tissue shadow and space between lateral incisor and premolar [Figure 3]. The case was provisionally diagnosed as irritational fibroma and differential diagnosis was pleomorphic adenoma.

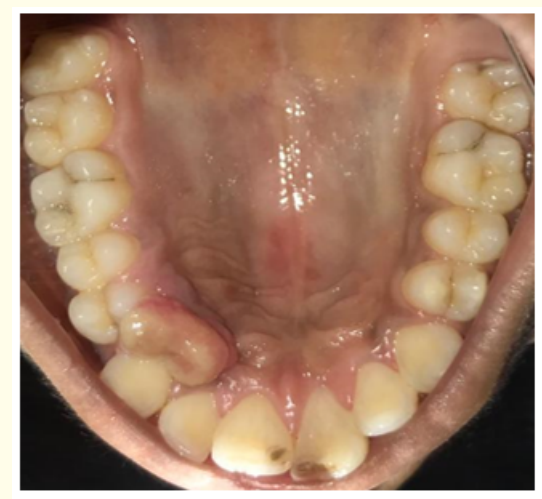

Figure 1 


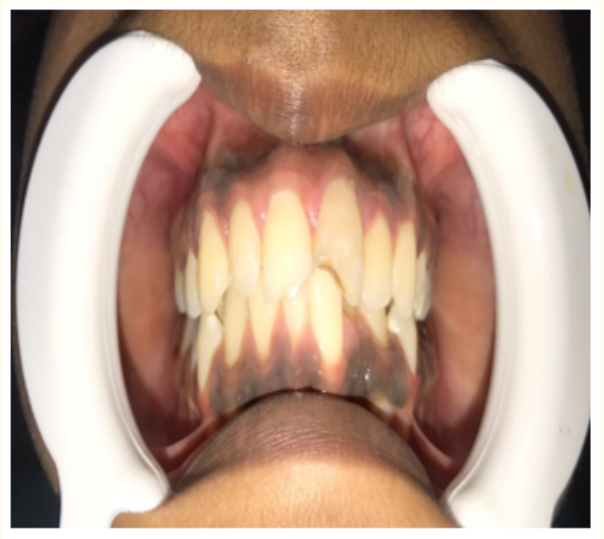

Figure 2

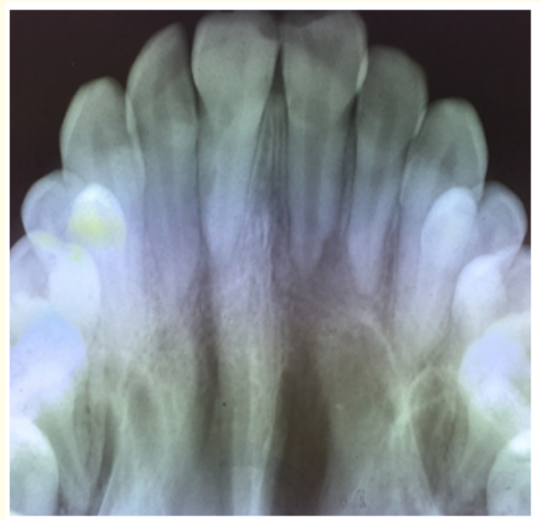

Figure 3

To avoid the risk of infective endocarditis paediatrician consent was taken. Clindamycin $600 \mathrm{mg}$ was prescribed as a single dose 1 hour before the procedure, because patient gave a history of allergic to penicillin. After routine blood examinations and consent of the patient excision of the lesion was done using NG: YAG diode laser emitting light at 1064 [Figure 4 and 5].

Histopathological examination revealed hyperplastic Para keratinized stratified squamous epithelium of irregular thickness [Figure7]. The underlying connective tissue is densely fibrous with haphazardly arranged collagen bundles interspersed with plump fibroblasts. stroma is densely infiltrated with diffuse inflammatory cell infiltrate predominantly of lymphocytes and plasma cells.
Blood vessels lined by endothelial cells are seen along with areas of haemorrhage. Areas of dystrophic calcification were also seen confirming the lesion as POF [Figure 8].

The follow up of the case showed normal healing of the area [Figure 9].

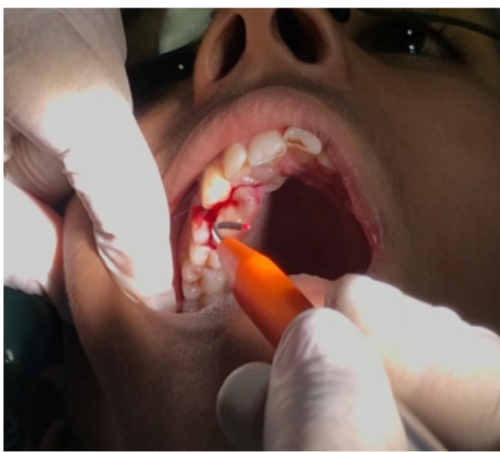

Figure 4

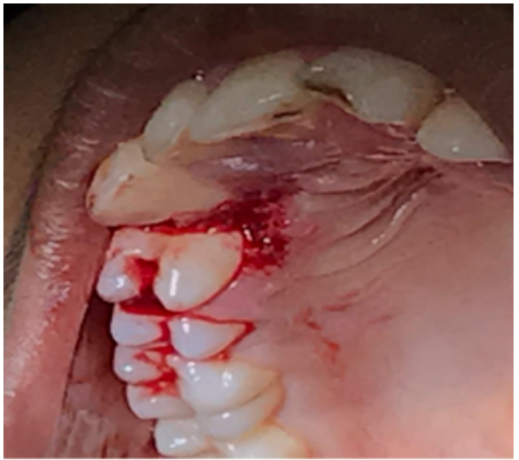

Figure 5

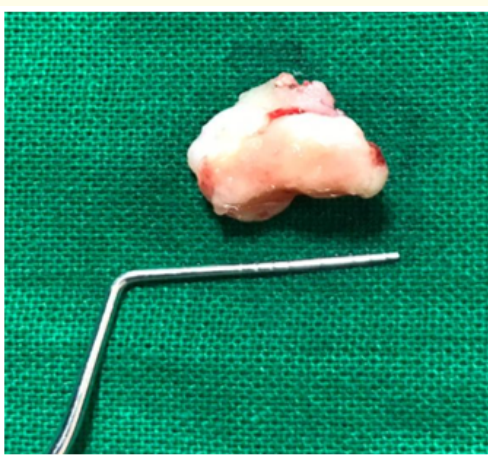

Figure 6: Excised tissue after laser surgery. 


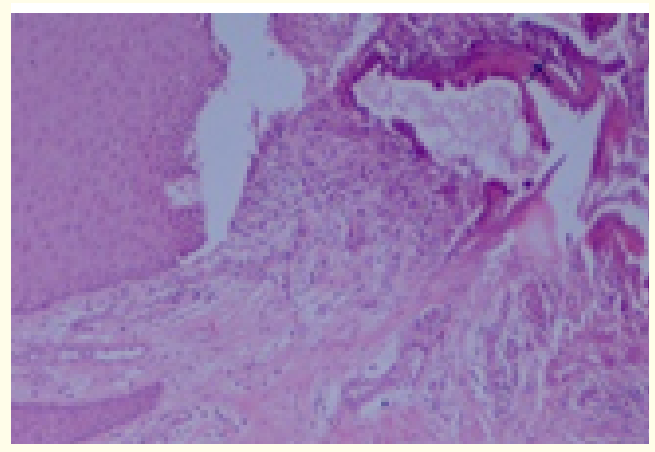

Figure 7

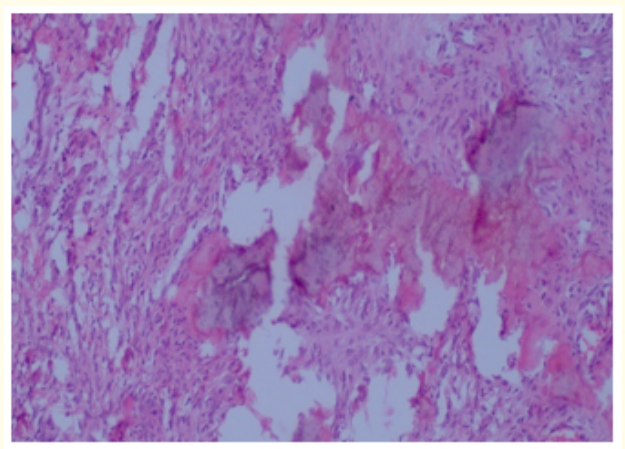

Figure 8

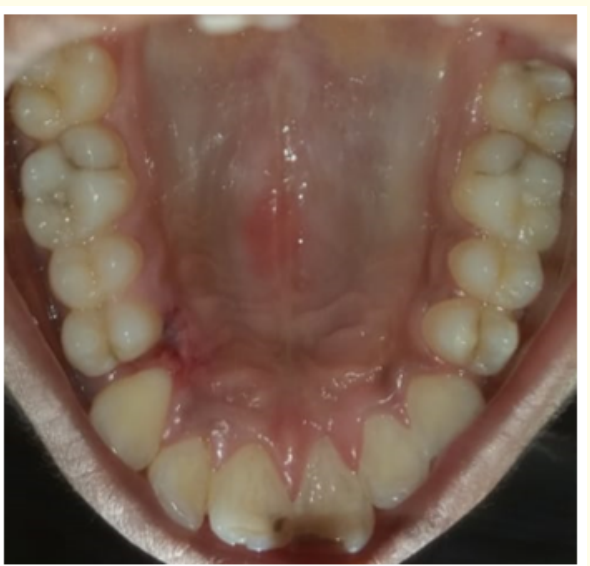

Figure 9: Normal healing after excision.

\section{Discussion}

POF is described by various synonyms such as peripheral cement ossifying fibroma, peripheral odontogenic fibroma (PODF) with cement genesis, peripheral fibroma with osteogenesis, peripheral fibroma with calcification, fibrous epulis, calcifying fibroblastic granuloma, etc. Other terms used in the past to describe this lesion were peripheral fibroma with calcification, ossifying fibrous epulis, and calcifying fibroblastic granuloma. It usually affects women in the second decade of life POF can be distinguished from neoplasm with significant growth potential. The color seems to be identical to that of the gingiva or slightly reddish and the surface may appear ulcerated $[1,2,4]$.

Almost $60 \%$ of the lesions occur in the maxilla and mostly found anterior to molars. Dental calculus, plaque, microorganisms, dental appliances, and restorations are considered to be the etiological factors triggering the lesion. The lesion though usually smaller than $1.5 \mathrm{~cm}$ in diameter can reach to a much larger size and can cause separation of the adjacent teeth, resorption of the alveolar crest, destruction of the bony structure and cosmetic deformity [1].

The definitive diagnosis is based on the histological examination, which depends on the identification of cellular connective tissue and the focal presence of bone or calcifications. There are two schools of thought proposed to understand the histogenesis of POF.

1. POF may initially develop as pyogenic granuloma that undergoes subsequent fibrous maturation and calcification. It represents the progressive stage of the same spectrum of pathos is.

2. POF is due to inflammatory hyperplasia of cells of periodontal ligament/periosteum. Metaplasia of the connective tissue leads to dystrophic calcification and bone formation $[3,4]$.

The differential diagnosis in this case was Irritational fibroma which is usually sessile, round or ovoid, nontender and may be lighter in colour than the surrounding tissue due to reduced vascularity. Due to the gradual and slow growth of the lesion, the patients are generally not aware of the mass. The irritational fibroma has a $66 \%$ female predilection and can occur at any age, but is usually seen in the 4 th to sixth decades of life. Contradicting to this evidence, this case presents an 15-year-old male child with the lesion. The presumed ethology was deep bite and trauma to the tissue [5]. 
A peripheral ossifying fibroma is usually small and does not require any further imaging study in addition to plain radiographs and histopathological diagnosis [6].

Histopathology shows no encapsulated mass of cellular connective tissue of mesenchymal origin characterized by fibrous connective tissue with varying fibroblast, myofibroblast, and collagen content, sparse to profuse endothelial proliferation. Mineralization can vary between cementum-like material, bone, and dystrophic calcification [8].

\section{Why usage of diode LASER for soft tissue excision?}

Diode NG: YAG laser is good for excising benign soft tissue lesions. Blood vessels smaller than $0.5 \mathrm{~mm}$ in diameter are sealed spontaneously, allowing excision visibility and precision when dissecting through the tissue. There is minimal cellular tissue damage adjacent to the plane of excision. This facilitates good wound healing, specimen can be removed without distortion. Laser excision is most desirable for any solid exophytic type lesion because of the improved visibility. precise control on tissue removal. Once the appropriate depth has been reached, excision can be performed by grasping the tissue with forceps, applying slight traction and horizontally undermining the tissue in the same fashion with the laser still in focused mode. There is minimal damage to the adjacent tissue and coagulation of denatured protein forms on the surface [10].

Treatment

The preferred management of POF is total excision. This should include the involved PDL and periosteum. Any etiology should be removed first, such as calculus, ill-fitted dental appliances, sharp cusps and rough restorations have to be removed. However, extraction of the adjacent teeth is seldom necessary in the early stages. Although POF is a benign and a reactive lesion, the recurrence rate is fairly high, that is upto $16 \%$, has been seen by studies [7].

Due to the high rate of recurrence ( $8 \%$ to $20 \%$ ), close postoperative monitoring is required in all cases of POF. POF reoccurs due to

1) The incomplete removal of the lesion

2) The failure to eliminate local irritants

3) Difficulty in accessing the extent of the lesion during surgical manipulation as a result of the intricate location of the lesion $[9,10]$.

\section{Conclusion}

Peripheral ossifying fibroma is a slowly progressing reactive lesion requiring complete removal of the lesions down to the periosteum and periodontal ligament along with regular post excision follow ups to minimize the possible chances of recurrence.

\section{Bibliography}

1. KS Poonacha., et al. "Peripheral ossifying fibroma: A clinical repor". Contempory Clinical Dentistry 1.1 (2010): 54-56.

2. Mergoni G., et al. "Peripheral ossifying fibroma: A clinicopathologic study of 27 cases and review of the literature with emphasis on histomorphologic features". Journal of Indian Society of Periodontology 19 (2015): 83-87.

3. Mohiuddin K., et al. "Peripheral ossifying fibroma". Journal of Indian Society of Periodontology 17 (2013): 507-509.

4. Mirchandani NM., et al. "Peripheral ossifying fibroma”. Journal of the International Clinical Dental Research Organisation 7 (2015): 159-161.

5. Mishra R., et al. "Pediatric Palatal Fibroma". International Journal of Clinical Pediatric Dentistry 10.1 (2017):96-98.

6. Swapnali Chaudhari and Hemant R Umarji. "Peripheral Ossifying Fibroma in the Oral Cavity: MRI Findings". Case Reports in Dentistry (2011).

7. Amberkar VS., et al. "Peripheral ossifying fibroma: Revisited". International Journal of Oral Health Sciences 7 (2017): 35-40.

8. Shah JS and Sharma S. "Peripheral ossifying fibroma: An unusual presentation". International Journal of Oral Health Sciences 8 (2018): 47-50.

9. Sumona Pal., et al. "The varying clinical presentations of peripheral ossifying fibroma: A report of three cases". The Revista Odonto Ciência 27.3 (2012): 251-255.

10. Di Ehsan Azma and Nassimeh Safavi. "Diode laser application in soft tissue oral surgery". Journal of Lasers in Medical Sciences 4.4 (2013): 206-211.

\section{Volume 3 Issue 11 November 2019}

(C) All rights are reserved by Amitha HA., et al. 